\title{
HOW TO RECOGNIZE POLYNOMIALS IN HIGHER ORDER SOBOLEV SPACES
}

\author{
BOGDAN BOJARSKI, LIZAVETA IHNATSYEVA and JUHA KINNUNEN*
}

\begin{abstract}
This paper extends characterizations of Sobolev spaces by Bourgain, Brézis, and Mironescu to the higher order case. As a byproduct, we obtain an integral condition for the Taylor remainder term, which implies that the function is a polynomial. Similar questions are also considered in the context of Whitney jets.
\end{abstract}

\section{Introduction}

In this paper we study a new characterization of the higher order Sobolev spaces $W^{m, p}(\Omega)$ which is based on J. Bourgain, H. Brézis, and P. Mironescu's approach [5] (see also [7]). They showed that a function $f \in L^{p}(\Omega)$ belongs to the first order Sobolev space $W^{1, p}(\Omega), 1<p<\infty$, on a smooth bounded domain $\Omega \subset \mathrm{R}^{n}$ if and only if

$$
\liminf _{\varepsilon \rightarrow 0} \int_{\Omega} \int_{\Omega} \frac{|f(x)-f(y)|^{p}}{|x-y|^{p}} \rho_{\varepsilon}(|x-y|) d x d y<\infty,
$$

where $\rho_{\varepsilon}$, with $\varepsilon>0$, are radial mollifiers. Moreover,

$$
\liminf _{\varepsilon \rightarrow 0} \int_{\Omega} \int_{\Omega} \frac{|f(x)-f(y)|^{p}}{|x-y|^{p}} \rho_{\varepsilon}(|x-y|) d x d y=c \int_{\Omega}|\nabla f|^{p} d x,
$$

where the constant $c$ depends only on $p$ and $n$. For $p=1$ this gives a characterization of the space of bounded variation $\operatorname{BV}(\Omega)$. See also [6], [8], [11], [16], [17], [18], [19], [22] and [21] for related results.

We extend the results of [5] and [7] to the higher order case. To characterize the Sobolev spaces $W^{m, p}(\Omega), 1<p<\infty$, we use the condition

$$
\liminf _{\varepsilon \rightarrow 0} \int_{\Omega} \int_{\Omega} \frac{\left|R^{m-1} f(x, y)\right|^{p}}{|x-y|^{m p}} \rho_{\varepsilon}(|x-y|) d x d y<\infty,
$$

\footnotetext{
* The research is supported by the Academy of Finland, the first author is also partially supported by Polish Ministry of Science grant no. N N201 397837 (years 2009-2012).

Received 28 March 2011.
} 
where $R^{m-1} f$ is a Taylor $(m-1)$-remainder of $f$, generalizing (1). For $p=1$ condition (2) describes the space $\mathrm{BV}^{m}(\Omega)$ of integrable functions whose weak derivatives of order $m$ are signed Radon measures with finite total variation.

Condition (2) is a priori weaker than the pointwise condition

$$
\left|R^{m-1} f(x, y)\right| \leq|x-y|^{m}\left(a_{f}(x)+a_{f}(y)\right), \quad a_{f} \in L^{p}\left(\mathrm{R}^{n}\right),
$$

characterizing Sobolev class $W^{m, p}\left(\mathrm{R}^{n}\right)$ as in [1] (see also (27) below).

Another variant of extension of the results of [5] and [7] to the higher order case has been introduced in [4], where the characterization of $W^{m, p}(\Omega), 1<$ $p<\infty,\left(\mathrm{BV}^{m}(\Omega)\right.$ for $\left.p=1\right)$ is formulated in terms of the $m$-th differences. According to this result, a function $f \in L^{p}(\Omega)$ belongs to $W^{m, p}(\Omega)$ if

(4) $\liminf _{\varepsilon \rightarrow 0} \int_{\Omega} \int_{\Omega} \mid \sum_{j=0}^{m}(-1)^{j}\left(\begin{array}{c}m \\ j\end{array}\right)$

$$
\left.f\left(\frac{(m-j)}{m} x+\frac{j}{m} y\right)\right|^{p} \frac{\rho_{\varepsilon}(|x-y|)}{|x-y|^{m p}} d x d y<\infty .
$$

For smooth functions $f \in C^{m+1}$ the equivalence of the integrands in (2) and (4) modulo $O\left(|x-y|^{m+1}\right)$ is well known. The results of the present paper and of [4] essentially show that both integrands are equivalent in their averaged asymptotic behaviour, for $\varepsilon \rightarrow 0$, in the $\varepsilon$-neighbourhood of the diagonal $\Delta=\{x=y\}$ in the Cartesian product $\Omega \times \Omega$.

In close connection with these characterizations, H. Brézis [7] considered conditions under which a measurable function $f$ defined on a connected open set $\Omega$ is a constant. See also [20]. In particular, he showed that if

$$
\int_{\Omega} \int_{\Omega} \frac{|f(x)-f(y)|}{|x-y|^{n+1}} d x d y<\infty,
$$

then $f$ is a constant function. We extend this result to the higher order case and show that the condition

$$
\int_{\Omega} \int_{\Omega} \frac{\left|R^{m-1} f(x, y)\right|}{|x-y|^{n+m}} d x d y<\infty
$$

implies that the function $f$, with locally integrable weak derivatives up to order $m-1$, is a polynomial of degree at most $m-1$.

Condition (2) applies to Whitney jets as well. Recall that H. Whitney in [24] gave a method to define differentiable functions on closed subsets of $\mathrm{R}^{n}$. His approach can be adopted to different kind of smoothness conditions. In particular, for an $(m-1)$-jet $F$ on a subset $\Omega \subset \mathrm{R}^{n}$, defined as a collection of 
functions $\left\{f_{\alpha}:|\alpha| \leq m-1\right\}, f_{\alpha} \in L^{p}(\Omega)$, we can study the meaning of (2) using the formal Taylor remainder of order $m-1$ of the jet $F$. The formalism of Taylor-Whitney jets identifies in a natural way virtual derivatives, jets, with Sobolev derivatives.

In Section 4 we show that a jet $F$ on an open set $\Omega$ satisfying (2) for some special case of mollifiers is locally a jet of a $W^{m, p}$-function. The proof uses an approximation procedure from [1] where the Sobolev spaces are described in terms of pointwise inequalities (3).

Notice also that a certain version of condition (2) for the jet $F$ on Ahlfors $s$-regular subsets $S \subset \mathrm{R}^{n}, n-1<s \leq n$, charaterizes the Lipschitz spaces $\operatorname{Lip}(m, p, \infty, S)$ studied in [13]. If $S$ supports the $q$-Poncaré inequality, $1 \leq$ $q<\infty$, then the first order space $\operatorname{Lip}(1, p, \infty, S)$ coincides with the HajłaszSobolev space $W^{1, p}(S)$ for $p>q$ (see e.g. [25]).

\section{Characterization of Sobolev spaces}

Our notation is standard. For a multi-index $\alpha=\left(\alpha_{1}, \ldots, \alpha_{n}\right), \alpha_{i} \geq 0$, and a point $x=\left(x_{1}, \ldots, x_{n}\right) \in \mathrm{R}^{n}$, we denote by

$$
x^{\alpha}=x_{1}^{\alpha_{1}} x_{2}^{\alpha_{2}} \cdots x_{n}^{\alpha_{n}}
$$

the monomial of degree

$$
|\alpha|=\sum_{i=1}^{n} \alpha_{i}
$$

In the same way

$$
D^{\alpha} f=\frac{\partial^{|\alpha|} f}{\partial x_{1}^{\alpha_{1}} \ldots \partial x_{n}^{\alpha_{n}}}
$$

is a (weak) partial derivative of order $|\alpha|$. We also use the convention that $D^{0} f=f$. Moreover, let $\nabla^{m} f$ be a vector with the components $D^{\alpha} f,|\alpha|=m$.

Let $\Omega$ be an open set in $\mathrm{R}^{n}, 1 \leq p<\infty, m$ a positive integer. The Sobolev space $W^{m, p}(\Omega)$ consists of all functions $u \in L^{p}(\Omega)$ such that for all multiindex $\alpha$ with $|\alpha| \leq m$ the weak derivative $D^{\alpha} u$ exists and belongs to $L^{p}(\Omega)$. We use the convention $W^{0, p}(\Omega)=L^{p}(\Omega)$. The Sobolev space $W^{m, p}(\Omega)$ is equipped with the norm

$$
\|u\|_{W^{m, p}(\Omega)}=\left(\sum_{|\alpha| \leq m} \int_{\Omega}\left|D^{\alpha} u\right|^{p} d x\right)^{1 / p} .
$$

For the properties of Sobolev functions, see [15]. 
We write

$$
T_{y}^{m} f(x)=\sum_{|\alpha| \leq m} D^{\alpha} f(y) \frac{(x-y)^{\alpha}}{\alpha !}
$$

and

$$
R^{m} f(x, y)=f(x)-T_{y}^{m} f(x)
$$

for the Taylor polynomial of order $m$ and the Taylor remainder of order $m$, respectively. If $f \in C^{m}\left(\mathrm{R}^{n}\right)$ the Taylor formula can be expressed in the following form, see [26] p. 126,

$$
\begin{aligned}
f(x) & -T_{y}^{m-1} f(x) \\
& \left.=m \sum_{|\alpha|=m}\left(\int_{0}^{1}(1-t)^{m-1} D^{\alpha} f[(1-t) y+t x)\right] d t\right) \frac{(x-y)^{\alpha}}{\alpha !} .
\end{aligned}
$$

Note that we use the same notation for formal Taylor polynomials and remainders if we have only weak derivatives.

We define a family of functions $\rho_{\varepsilon} \in L_{\mathrm{loc}}^{1}(0, \infty), \varepsilon>0$, such that $\rho_{\varepsilon} \geq 0$,

$$
\int_{0}^{\infty} \rho_{\varepsilon}(r) r^{n-1} d r=1
$$

and

$$
\lim _{\varepsilon \rightarrow 0} \int_{\delta}^{\infty} \rho_{\varepsilon}(r) r^{n-1} d r=0 \quad \text { for every } \quad \delta>0 .
$$

These properties are rather standard in the construction of radial mollifiers related to approximations of unity.

First we prove a useful result for smooth functions.

LeMmA 2.1. Let $\Omega$ be an open set in $\mathrm{R}^{n}, 1 \leq p<\infty$, $m$ a positive integer and $g \in C_{0}^{m+1}\left(\mathrm{R}^{n}\right)$. Then

(6) $\lim _{\varepsilon \rightarrow 0} \int_{\Omega} \int_{\Omega} \frac{\left|R^{m-1} g(x, y)\right|^{p}}{|x-y|^{m p}} \rho_{\varepsilon}(|x-y|) d x d y$

$$
=\int_{\Omega} \int_{\partial B(0,1)}\left|\sum_{|\alpha|=m} \frac{D^{\alpha} g(x)}{\alpha !} e^{\alpha}\right|^{p} d e d x .
$$

Proof. Since $g \in C_{0}^{m+1}\left(\mathrm{R}^{n}\right)$ by Taylor's formula, we have

$$
\left|R^{m-1} g(x+h, x)\right| \leq\left|\sum_{|\alpha|=m} \frac{D^{\alpha} g(x)}{\alpha !} h^{\alpha}\right|+c^{\prime}|h|^{m+1}
$$


from which we conclude that

$$
\left|R^{m-1} g(x+h, x)\right|^{p} \leq(1+\theta)\left|\sum_{|\alpha|=m} \frac{D^{\alpha} g(x)}{\alpha !} h^{\alpha}\right|^{p}+c_{\theta}^{\prime}|h|^{(m+1) p}
$$

for every $\theta>0, x \in \mathrm{R}^{n}$ and $h \in \mathrm{R}^{n}$.

We multiply the last inequality by $\rho_{\varepsilon}(|h|) /|h|^{m p}$ and integrate over the set

$$
\begin{aligned}
S=\{(x, h) \in( & \left.\operatorname{supp} g \cap \Omega) \times \mathrm{R}^{n}: x+h \in \Omega\right\} \\
& \cup\left\{(x, h) \in(\Omega \backslash \operatorname{supp} g) \times \mathrm{R}^{n}: x+h \in(\operatorname{supp} g \cap \Omega)\right\} .
\end{aligned}
$$

We have

$$
\begin{aligned}
& \iint_{S} \frac{\left|R^{m-1} g(x+h, x)\right|^{p}}{|h|^{m p}} \rho_{\varepsilon}(|h|) d h d x \\
& \leq(1+\theta) \int_{\Omega} \int_{\mathrm{R}^{n}} \frac{\rho_{\varepsilon}(|h|) \mid}{|h|^{m p}}\left|\sum_{|\alpha|=m} \frac{D^{\alpha} g(x)}{\alpha !} h^{\alpha}\right|^{p} d h d x \\
&+2 c_{\theta}^{\prime}|\operatorname{supp} g| \int_{\mathrm{R}^{n}}|h|^{p} \rho_{\varepsilon}(|h|) d h .
\end{aligned}
$$

By the properties of the mollifiers $\rho_{\varepsilon}$, it follows that

$$
\lim _{\varepsilon \rightarrow 0} \int_{\mathrm{R}^{n}}|h|^{p} \rho_{\varepsilon}(|h|) d h=0 .
$$

Note also, that

$$
\begin{aligned}
\int_{\mathrm{R}^{n}} \frac{\rho_{\varepsilon}(|h|)}{|h|^{m p}}\left|\sum_{|\alpha|=m} \frac{D^{\alpha} g(x)}{\alpha !} h^{\alpha}\right|^{p} d h & \\
& =\int_{0}^{\infty} r^{n-1} \rho_{\varepsilon}(r) d r \int_{\partial B(0,1)}\left|\sum_{|\alpha|=m} \frac{D^{\alpha} g(x)}{\alpha !} e^{\alpha}\right|^{p} d e .
\end{aligned}
$$

Thus, passing to the limit in (7), first with $\varepsilon \rightarrow 0$, then with $\theta \rightarrow 0$, and changing variables in the integral on the left hand side, we arrive at

(9) $\quad \limsup _{\varepsilon \rightarrow 0} \int_{\Omega} \int_{\Omega} \frac{\left|R^{m-1} g(x, y)\right|^{p}}{|x-y|^{m p}} \rho_{\varepsilon}(|x-y|) d x d y$

$$
\leq \int_{\Omega} \int_{\partial B(0,1)}\left|\sum_{|\alpha|=m} \frac{D^{\alpha} g(x)}{\alpha !} e^{\alpha}\right|^{p} d e d x .
$$

This concludes the first part of the proof of (6). 
Next we show the inequality to the other direction. If $K$ is a compact subset of $\Omega$, then for any $x \in K$ and $|h| \leq d$, where

$$
d=\min \{1, \operatorname{dist}(K, \partial \Omega) / 2\},
$$

we have

$$
\left|R^{m-1} g(x+h, x)-\sum_{|\alpha|=m} \frac{D^{\alpha} g(x)}{\alpha !} h^{\alpha}\right| \leq c_{K}|h|^{m+1} .
$$

Hence

$$
\left|\sum_{|\alpha|=m} \frac{D^{\alpha} g(x)}{\alpha !} h^{\alpha}\right|^{p} \leq(1+\theta)\left|R^{m-1} g(x+h, x)\right|^{p}+c_{\theta, K}|h|^{(m+1) p}
$$

for every $\theta>0$ and consequently

$$
\begin{aligned}
& \int_{K} \int_{B(0, d)} \frac{\rho_{\varepsilon}(|h|)}{|h|^{m p}}\left|\sum_{|\alpha|=m} \frac{D^{\alpha} g(x)}{\alpha !} h^{\alpha}\right|^{p} d h d x \\
& \leq(1+\theta) \int_{K} \int_{B(0, d)} \frac{\left|R^{m-1} g(x+h, x)\right|^{p}}{|h|^{m p}} \rho_{\varepsilon}(|h|) d h d x \\
& \quad+c_{\theta, K}|K| \int_{B(0, d)}|h|^{p} \rho_{\varepsilon}(|h|) d h
\end{aligned}
$$

Passing to the limit as $\varepsilon \rightarrow 0$ in (10), and taking into account (8), we have

$$
\begin{aligned}
& \int_{K} \int_{\partial B(0,1)}\left|\sum_{|\alpha|=m} \frac{D^{\alpha} g(x)}{\alpha !} e^{\alpha}\right|^{p} d e d x \\
& \leq(1+\theta) \liminf _{\varepsilon \rightarrow 0} \int_{K} \int_{B(0, d)} \frac{\left|R^{m-1} g(x+h, x)\right|^{p}}{|h|^{m p}} \rho_{\varepsilon}(|h|) d h d x \\
& \quad \leq(1+\theta) \liminf _{\varepsilon \rightarrow 0} \int_{\Omega} \int_{\Omega} \frac{\left|R^{m-1} g(x, y)\right|^{p}}{|x-y|^{m p}} \rho_{\varepsilon}(|x-y|) d x d y .
\end{aligned}
$$

Since the last estimate holds for every $\theta>0$ and every compact set $K \subset \Omega$, we have

$$
\begin{aligned}
\int_{\Omega} \int_{\partial B(0,1)} \mid \sum_{|\alpha|=m} & \left.\frac{D^{\alpha} g(x)}{\alpha !} e^{\alpha}\right|^{p} d e d x \\
& \leq \liminf _{\varepsilon \rightarrow 0} \int_{\Omega} \int_{\Omega} \frac{\left|R^{m-1} g(x, y)\right|^{p}}{|x-y|^{m p}} \rho_{\varepsilon}(|x-y|) d x d y
\end{aligned}
$$


Combining this with (9) we arrive at (6).

The following theorem is an analog of Theorem 2 in [7] (see also [5]) for higher order Sobolev spaces.

Theorem 2.2. Let $\Omega$ be an open set in $\mathrm{R}^{n}, 1<p<\infty$ and $m$ be a positive integer. If $f \in W^{m-1, p}(\Omega)$ satisfies

$$
c_{f}=\liminf _{\varepsilon \rightarrow 0} \int_{\Omega} \int_{\Omega} \frac{\left|R^{m-1} f(x, y)\right|^{p}}{|x-y|^{m p}} \rho_{\varepsilon}(|x-y|) d x d y<\infty,
$$

then $f \in W^{m, p}(\Omega)$.

Proof. Assume that $\Omega^{\prime} \Subset \Omega$ and $\delta<\operatorname{dist}\left(\Omega^{\prime}, \partial \Omega\right)$. Let $\eta \in C_{0}^{\infty}\left(\mathrm{R}^{n}\right)$ be a nonnegative radial function such that

$$
\int_{\mathrm{R}^{n}} \eta(x) d x=1
$$

and supp $\eta \subset B(0,1)$. Consider the regularization $f_{\delta}=f * \eta_{\delta}$ of $f, \eta_{\delta}(x)=$ $\delta^{-n} \eta(x / \delta)$. For every $f \in L_{\text {loc }}^{1}(\Omega)$ (extended by zero to $\mathrm{R}^{n} \backslash \Omega$ ) the function $f_{\delta}$ is smooth in $\Omega$, and if $f$ has a weak derivative $D^{\alpha} f$ in $\Omega^{\prime}$, then

$$
D^{\alpha}\left(f_{\delta}\right)=D^{\alpha} f * \eta_{\delta}
$$

(see e.g. [15]). Thus, for every $x, y \in \Omega^{\prime}$ we have

$$
\begin{aligned}
R^{m-1} f_{\delta}(x, y) & =f_{\delta}(x)-\sum_{|\alpha| \leq m-1} D^{\alpha}\left(f_{\delta}\right)(y) \frac{(x-y)^{\alpha}}{\alpha !} \\
& =\int_{B(0, \delta)}\left(f(x-z)-\sum_{|\alpha| \leq m-1} D^{\alpha} f(y-z) \frac{(x-y)^{\alpha}}{\alpha !}\right) \eta_{\delta}(z) d z \\
& =\int_{B(0, \delta)} R^{m-1} f(x-z, y-z) \eta_{\delta}(z) d z .
\end{aligned}
$$

By Jensen's inequality, it is easy to see that (12) implies

$$
\liminf _{\varepsilon \rightarrow 0} \int_{\Omega^{\prime}} \int_{\Omega^{\prime}} \frac{\left|R^{m-1} f_{\delta}(x, y)\right|^{p}}{|x-y|^{m p}} \rho_{\varepsilon}(|x-y|) d x d y \leq c_{f} .
$$

Next by applying (11) to $g=f_{\delta}$ we get

$$
\int_{\Omega^{\prime}} \int_{\partial B(0,1)}\left|\sum_{|\alpha|=m} \frac{D^{\alpha} f_{\delta}(x)}{\alpha !} e^{\alpha}\right|^{p} d e d x \leq c_{f} .
$$


Now let $e \in \partial B(0,1)$ and denote by $E$ the vector with the components $E_{\alpha}=(1 / \alpha !) e_{1}^{\alpha_{1}} \cdots e_{n}^{\alpha_{n}},|\alpha|=m$. It is easy to see that

$$
\|v\|=\left(\int_{\partial B(0,1)}|v \cdot E|^{p} d e\right)^{1 / p}, \quad 1 \leq p<\infty,
$$

is a norm on a linear space of all vectors $v=\left(v_{\alpha}\right)_{|\alpha|=m}$. Obviously, it is nonnegative and $\|v\|=0$ if and only if $v=0$. The triangle inequality follows from the Minkowski inequality. Since $\|\cdot\|$ is equivalent to the Euclidean norm, for any vector $\nabla^{m} g(x)$ we have

$$
\left|\nabla^{m} g(x)\right|^{p} \approx \int_{\partial B(0,1)}\left|\nabla^{m} g(x) \cdot E\right|^{p} d e
$$

and (14) implies that

$$
\int_{\Omega^{\prime}}\left|\nabla^{m} f_{\delta}(x)\right|^{p} d x \leq c
$$

for every $\Omega^{\prime} \Subset \Omega$ and $\delta<\operatorname{dist}\left(\Omega^{\prime}, \partial \Omega\right)$, with the constant $c$ depending only on $c_{f}, n, m$ and $p$.

Since $p>1$ the weak compactness and a diagonal argument show that $f \in W^{m, p}(\Omega)$.

The following statement follows immediately from Theorem 2.2.

Corollary 2.3. Let $\Omega$ be an open set in $\mathrm{R}^{n}, 1<p<\infty, m$ a positive integer. If $f \in L^{p}(\Omega)$ satisfies

$$
\liminf _{\varepsilon \rightarrow 0} \int_{\Omega} \int_{\Omega} \frac{\left|R^{i-1} f(x, y)\right|^{p}}{|x-y|^{i p}} \rho_{\varepsilon}(|x-y|) d x d y<\infty,
$$

for every $i=1, \ldots, m$, then $f \in W^{m, p}(\Omega)$.

Here the remainders $R^{i-1} f$ are defined recursively starting from $i=1$. Thus, if (17) holds for $i=1$ by Theorem 2.2 the weak derivatives $D^{\alpha} f$, $|\alpha|=1$, exist and $R^{i-1} f$ are defined for $i=2$ and this procedure can be continued recursively. The recursion may seem somewhat awckward. A more direct interpretation is possible in terms of Whitney jets, see Section 4.

REMARK 2.4. Note that if $\Omega$ is a $W^{m, p}$-extension domain, i.e. there is a bounded linear operator

$$
\mathscr{E}: W^{m, p}(\Omega) \rightarrow W^{m, p}\left(\mathrm{R}^{n}\right)
$$


such that $\left.\mathscr{E} f\right|_{\Omega}=f$ for every $f \in W^{m, p}(\Omega)$, then also the converse statement in Theorem 2.2 is true. Indeed, if $f \in W^{m, p}\left(\mathrm{R}^{n}\right)$, then

$$
\int_{\mathrm{R}^{n}}\left|R^{m-1} f(x+h, x)\right|^{p} d x \leq c|h|^{m p} \int_{\mathrm{R}^{n}}\left|\nabla^{m} f(x)\right|^{p} d x
$$

for every $h \in \mathrm{R}^{n}$. For smooth functions inequality (18) follows from Taylor's formula (5), thus we have

$$
R^{m-1} f(x+h, x)=m \sum_{|\alpha|=m} \frac{h^{\alpha}}{\alpha !} \int_{0}^{1}(1-t)^{m-1} D^{\alpha} f(x+t h) d t .
$$

And an integration over the whole space gives

$$
\begin{aligned}
\int_{\mathrm{R}^{n}} \mid & \left.R^{m-1} f(x+h, x)\right|^{p} d x \\
& \leq c|h|^{m p} \int_{\mathrm{R}^{n}} \int_{0}^{1}(1-t)^{(m-1) p}\left|\nabla^{m} f(x+t h)\right|^{p} d t d x \\
& \leq c|h|^{m p} \int_{0}^{1} \int_{\mathrm{R}^{n}}\left|\nabla^{m} f(x+t h)\right|^{p} d x d t \\
& \leq c|h|^{m p} \int_{\mathrm{R}^{n}}\left|\nabla^{m} f(x)\right|^{p} d x .
\end{aligned}
$$

Since smooth functions are dense in the Sobolev space inequality (18) holds for every $f \in W^{m, p}\left(\mathrm{R}^{n}\right)$.

Now let $f \in W^{m, p}(\Omega)$ and denote by $\tilde{f}$ its extension to $\mathrm{R}^{n}$. Since

$$
\int_{\mathrm{R}^{n}} \rho_{\varepsilon}(|h|) d h=\omega_{n-1} \int_{0}^{\infty} \rho_{\varepsilon}(r) r^{n-1} d r=\omega_{n-1},
$$

where $\omega_{n-1}$ is the $(n-1)$-dimensional surface measure of the unit ball in $\mathrm{R}^{n}$, by (18) we have

$$
\begin{aligned}
& \int_{\Omega} \int_{\Omega} \frac{\left|R^{m-1} f(x, y)\right|^{p}}{|x-y|^{m p}} \rho_{\varepsilon}(|x-y|) d y d x \\
& \quad \leq \int_{\mathrm{R}^{n}} \int_{\mathrm{R}^{n}} \frac{\left|R^{m-1} \tilde{f}(x, y)\right|^{p}}{|x-y|^{m p}} \rho_{\varepsilon}(|x-y|) d y d x \\
& \quad \leq c \int_{\mathrm{R}^{n}}\left|\nabla^{m} \tilde{f}(x)\right|^{p} d x \\
& \quad \leq c\|\tilde{f}\|_{W^{m, p}\left(\mathbb{R}^{n}\right)} \leq c\|f\|_{W^{m, p}(\Omega)} .
\end{aligned}
$$


More precisely, the following result is true.

THEOREM 2.5. Assume that $\Omega$ is a $W^{m, p}$-extension domain, let $m$ be a positive integer, $1<p<\infty$ and let $f \in W^{m, p}(\Omega)$. Then

$$
\begin{aligned}
\lim _{\varepsilon \rightarrow 0} \int_{\Omega} \int_{\Omega} \frac{\left|R^{m-1} f(x, y)\right|^{p}}{|x-y|^{m p}} & \rho_{\varepsilon}(|x-y|) d x d y \\
& =\int_{\Omega} \int_{\partial B(0,1)}\left|\sum_{|\alpha|=m} \frac{D^{\alpha} f(x)}{\alpha !} e^{\alpha}\right|^{p} d e d x .
\end{aligned}
$$

Proof. Since $\Omega$ is an extension domain, any function $f \in W^{m, p}(\Omega)$ can be approximated by functions $f_{n} \in C_{0}^{m+1}\left(\mathrm{R}^{n}\right)$ in $W^{m, p}(\Omega)$-norm. Hence, Lemma 2.1 implies the validity of (6) for every $f \in W^{m, p}(\Omega)$. Indeed, by (15) for the right hand side of (6) we have

$$
\begin{aligned}
& \left|\left(\int_{\partial B(0,1)}\left|\sum_{|\alpha|=m} \frac{D^{\alpha} f(x)}{\alpha !} e^{\alpha}\right|^{p} d e\right)^{1 / p}-\left(\int_{\partial B(0,1)}\left|\sum_{|\alpha|=m} \frac{D^{\alpha} f_{n}(x)}{\alpha !} e^{\alpha}\right|^{p} d e\right)^{1 / p}\right| \\
& =\left|\left\|\nabla^{m} f(x)\right\|-\left\|\nabla^{m} f_{n}(x)\right\|\right| \leq\left\|\nabla^{m}\left(f-f_{n}\right)(x)\right\| \leq c\left|\nabla^{m}\left(f-f_{n}\right)(x)\right| .
\end{aligned}
$$

To justify the limit of the left hand side of (6) we can apply (19). Thus, equality (6) is true for any $f \in W^{m, p}(\Omega)$.

REMARK 2.6. If $\Omega=\mathrm{R}^{n}$ and

$$
\rho_{\varepsilon}(r)= \begin{cases}\frac{(n+m p) r^{m p}}{\varepsilon^{n+m p}}, & r<\varepsilon, \\ 0, & r \geq \varepsilon,\end{cases}
$$

then (12) can be written as

$$
\liminf _{\varepsilon \rightarrow 0} \frac{1}{\varepsilon^{m p}} \int_{\mathrm{R}^{n}} f_{B(y, \varepsilon)}\left|f(x)-T_{y}^{m-1} f(x)\right|^{p} d x d y<\infty .
$$

Here the integral sign with a bar denotes the integral average.

We point out that condition (22) is closely related to Calderón's characterization of Sobolev spaces in [9] (see also [12]). To this end, let $1<p<\infty$ and $m$ be a positive integer. For $f \in L^{p}(\Omega)$ we define a maximal function as

$$
\mathcal{N}(f, y)=\sup _{\varepsilon>0} \frac{1}{\varepsilon^{m}}\left(f_{B(y, \varepsilon)}|f(x)-P(x, y)|^{p} d x\right)^{1 / p},
$$

if there exists a polynomial $P(x, y)$ in $x$, of degree at most $m-1$, such that the expression on the right hand side of (23) is finite. If no such polynomial exists, 
we set $\mathcal{N}(f, y)=\infty$. Then a function $f \in L^{p}\left(\mathrm{R}^{n}\right)$ belongs to $W^{m, p}\left(\mathrm{R}^{n}\right)$ if and only if $\mathcal{N}(f, \cdot) \in L^{p}\left(\mathrm{R}^{n}\right)$.

Now consider the analog of Theorem 2.2 for $p=1$. Recall that the space $\operatorname{BV}(\Omega)$ is defined as the space of functions in $L^{1}(\Omega)$, whose weak derivatives are Radon measures with finite total variation. Denote by $\mathrm{BV}^{m}(\Omega)$, $m=2,3, \ldots$, the set of functions in $L^{1}(\Omega)$, whose derivatives of order $m$ are finite Radon measures.

Observe, that by the Riesz representation theorem, a function $f \in L^{1}(\Omega)$ belongs to $\mathrm{BV}^{m}(\Omega)$ if and only if there exists $c>0$ such that

$$
\left|\int_{\Omega} f D^{\alpha} \varphi d x\right| \leq c\|\varphi\|_{\infty}
$$

for every $\varphi \in C_{0}^{\infty}(\Omega)$ and every multi-index $\alpha$ with $|\alpha|=m$.

THEOREM 2.7. Let $\Omega$ be an open set in $\mathrm{R}^{n}$ and $m$ be a positive integer. If $f \in W^{m-1,1}(\Omega)$ satisfies

$$
\liminf _{\varepsilon \rightarrow 0} \int_{\Omega} \int_{\Omega} \frac{\left|R^{m-1} f(x, y)\right|}{|x-y|^{m}} \rho_{\varepsilon}(|x-y|) d x d y<\infty,
$$

then $f \in \mathrm{BV}^{m}(\Omega)$.

Proof. The proof is the same as for Theorem 2.2, except for the fact that (16) implies that all the derivatives of order $m$ of function $f$ are measures with finite total variation.

REMARK 2.8. As observed in [4], if $\Omega$ is a smooth bounded domain, then $\mathrm{BV}^{m}(\Omega)$ can be characterized as the set of functions $f \in W^{m-1,1}(\Omega)$ such that $D^{\alpha} f \in \operatorname{BV}(\Omega)$ for every multi-index $\alpha$ with $|\alpha|=m-1$. The equivalence follows from the Sobolev embedding theorem, see e.g. [15].

Using this characterization it is not difficult to see that Theorem 2.7 gives a necessary and sufficient condition for a function to be in $\mathrm{BV}^{m}(\Omega)$, when $\Omega$ is a bounded smooth domain in $\mathrm{R}^{n}$. This can be seen as in Remark 2.4.

\section{A criterion for a function to be a polynomial}

The next result is a higher order version of Theorem 1 in [7].

THeOREM 3.1. Let $\Omega$ be a connected open set in $\mathrm{R}^{n}, 1 \leq p<\infty$ and $m$ a positive integer. Assume that $f \in L_{\mathrm{loc}}^{1}(\Omega)$ has weak derivatives $D^{\alpha} f$ with $|\alpha| \leq m-1$ in $\Omega$ and

$$
\liminf _{\varepsilon \rightarrow 0} \int_{\Omega} \int_{\Omega} \frac{\left|R^{m-1} f(x, y)\right|^{p}}{|x-y|^{m p}} \rho_{\varepsilon}(|x-y|) d x d y=0 .
$$

Then $f$ is a polynomial of degree at most $m-1$ a.e. on $\Omega$. 
Proof. Suppose first that $p>1$. Note that if $\Omega$ is a bounded smooth domain in Theorem 2.2 it is enough to assume that $f \in L_{\text {loc }}^{1}(\Omega)$ has weak derivatives $D^{\alpha} f$ with $|\alpha| \leq m-1$ in $\Omega$. Thus, applying Theorem 2.2 (see also Theorem 2.5) to a ball $B \subset \Omega$ we can conclude that $f \in W^{m, p}(B)$ and $\left\|\nabla^{m} f\right\|_{L^{p}(B)}=0$. This implies that $f$ is a polynomial of degree at most $m-1$ a.e. in $B$ (see e.g. generalized Poincaré inequality in [15]) and the claim follows from the assumption that $\Omega$ is connected.

Let then $p=1$. In this case we apply Theorem 2.7 to a ball $B \subset \Omega$ and conclude that $f \in \mathrm{BV}^{m}(B)$ and the total variation $\left|\nabla^{m} f\right|(B)$ of the vector valued measure $\nabla^{m} f$ equal to zero. This implies that there is a polynomial $P$ of degree at most $m-1$ such that $f=P$ a.e. in $B$ (see e.g. Lemma 12 in [4] for more details). Again the claim follows from the assumption that $\Omega$ is connected.

The next result is a higher order generalization of Proposition 1 in [7].

COROLlaRy 3.2. Let $\Omega$ be a connected open set in $\mathrm{R}^{n}, 1 \leq p<\infty$ and $m$ a positive integer. Suppose that $f \in L_{\mathrm{loc}}^{1}(\Omega)$ has weak derivatives $D^{\alpha} f$ with $|\alpha| \leq m-1$ in $\Omega$ and

$$
\int_{\Omega} \int_{\Omega} \frac{\left|R^{m-1} f(x, y)\right|^{p}}{|x-y|^{m p+n}} d x d y<\infty .
$$

Then $f$ is a polynomial of degree at most $m-1$ a.e. on $\Omega$.

Proof. By choosing

$$
\rho_{\varepsilon}(r)= \begin{cases}\varepsilon r^{-n+\varepsilon}, & r<1, \\ 0, & r \geq 1,\end{cases}
$$

we have

$$
\begin{aligned}
& \liminf _{\varepsilon \rightarrow 0} \varepsilon \int_{\Omega} \int_{\Omega} \frac{\left|R^{m-1} f(x, y)\right|^{p}}{|x-y|^{m p+n-\varepsilon}} d x d y \\
& \quad \leq \liminf _{\varepsilon \rightarrow 0} \varepsilon \int_{\Omega} \int_{\Omega} \frac{\left|R^{m-1} f(x, y)\right|^{p}}{|x-y|^{m p+n}} d x d y=0 .
\end{aligned}
$$

The claim follows from Theorem 3.1.

Again there is an interpretation of the previous result in terms of Whitney jets. Indeed, it is possible to state the corollary for Whitney jets without referring to lower order derivatives, see Remark 4.2. 


\section{Whitney jets}

In this section we show that a jet of functions, whose formal Taylor remainder satisfies (22), or its counterpart on a subdomain, can be identified with the jet of weak derivatives of a Sobolev function.

First we recall terminology related to the Whitney jet theory. Assume that $\Omega$ is an open set in $\mathrm{R}^{n}$ and let $m$ be a positive integer. An $m$-jet $F \in J^{m}(\Omega)$ is a collection

$$
\left\{f_{\alpha}:|\alpha| \leq m\right\}
$$

of functions. The $m$-jets define the formal Taylor polynomials in $x \in \mathrm{R}^{n}$ (centered at $y \in \Omega$ )

$$
T_{y}^{k} F(x)=\sum_{|\alpha| \leq k} f_{\alpha}(y) \frac{(x-y)^{\alpha}}{\alpha !},
$$

with $k \leq m$, and

$$
T_{y, j}^{k-|j|} F(x)=\sum_{|j+\alpha| \leq k} f_{j+\alpha}(y) \frac{(x-y)^{\alpha}}{\alpha !},
$$

with $|j| \leq k \leq m$. The formal Taylor remainders are defined to be

$$
R^{k} F(x, y)=f_{0}(x)-T_{y}^{k} F(x)
$$

and

$$
R_{j}^{k-|j|} F(x, y)=f_{j}(x)-T_{y, j}^{k-|j|} F(x),
$$

where $x, y \in K$ and $|j| \leq k \leq m$.

Let $Q$ be a fixed cube in $\mathrm{R}^{n}$, an $(m-1)$-jet $F \in J^{m-1}(Q)$,

$$
F=\left\{f_{j}:|j| \leq m-1\right\},
$$

where $f_{j} \in L^{p}(Q)$, is said to be an $(m-1)$-jet in $Q$ with variable Lipchitz coefficients, denoted by $F \in \operatorname{VLC}(m, p, Q)$, if the pointwise inequality

$$
\left|R^{m-1} F(x, y)\right| \leq|x-y|^{m}\left(a_{Q}(x)+a_{Q}(y)\right),
$$

where $x, y \in Q$, holds for some function $a_{Q}=a_{Q}(F) \in L^{p}(Q)$.

The $(m-1)$-jet spaces $\operatorname{VLC}(m, p, Q)$ have been studied in [1] and it has been shown that $\operatorname{VLC}(m, p, Q)$ regarded as a Banach space and equipped with the norm

$$
\|F\|=\max \left\{\left\|f_{j}\right\|_{L^{p}(Q)}:|j| \leq m-1\right\}+\inf \left\|a_{Q}\right\|_{L^{p}(Q)},
$$


can be identified with the classical Sobolev space $W^{m, p}(Q)$. The fact that inequality (27) holds for a function $f \in W^{m, p}(Q)$ has been proved before in [3].

Let us consider an $(m-1)$-jet $F \in J^{m-1}(\Omega)$ of locally integrable functions on an open set $\Omega$ in $\mathrm{R}^{n}$ with the property that its formal Taylor remainder of order $m-1$ satisfies the condition

$$
a_{F}=\lim _{\varepsilon \rightarrow 0} \frac{1}{\varepsilon^{n+m p}} \iint_{\{(x, y) \in \Omega:|x-y|<\varepsilon\}}\left|R^{m-1} F(x, y)\right|^{p} d x d y<\infty .
$$

This is a special case of condition (12) with mollifiers (21).

Note that pointwise estimate (27) for $x, y \in \Omega$ implies (28). Indeed,

$$
\begin{aligned}
\int_{\Omega} \int_{\Omega} & \left|R^{m-1} F(x, y)\right|^{p} \chi_{\{|x-y|<\varepsilon\}} d x d y \\
& \leq \int_{\Omega} \int_{\Omega}|x-y|^{m p} \chi_{\{|x-y|<\varepsilon\}}\left(a_{\Omega}(x)+a_{\Omega}(y)\right)^{p} d x d y \\
& \leq c \int_{\Omega} \int_{\Omega}|x-y|^{m p} \chi_{\{|x-y|<\varepsilon\}} a_{\Omega}^{p}(x) d x d y
\end{aligned}
$$

and we have

$$
\begin{aligned}
& \frac{1}{\varepsilon^{n+m p}} \iint_{\{(x, y) \in \Omega:|x-y|<\varepsilon\}}\left|R^{m-1} F(x, y)\right|^{p} d x d y \\
& \quad \leq c \int_{\Omega} a_{\Omega}^{p}(x) \int_{\Omega} \frac{|x-y|^{m p}}{\varepsilon^{n+m p}} \chi_{\{|x-y|<\varepsilon\}} d y d x \\
& \quad \leq c \frac{\omega_{n-1}}{n}\left\|a_{\Omega}\right\|_{L^{p}(\Omega)}^{p} .
\end{aligned}
$$

At the same time (28) is a sufficient condition for a jet to be identified with a Sobolev function. More precisely, the following theorem holds true.

THEOREM 4.1. Let $\Omega$ be an open set in $\mathrm{R}^{n}, 1<p<\infty$ and $m$ be a positive integer. Assume that an $(m-1)$-jet

$$
F=\left\{f_{\alpha}:|\alpha| \leq m-1\right\},
$$

where $f_{\alpha} \in L^{p}(\Omega)$, satisfies condition (28). Then for every $\Omega^{\prime} \Subset \Omega$ there is a function $f \in W^{m, p}\left(\Omega^{\prime}\right)$ such that

$$
\left.f_{\alpha}\right|_{\Omega^{\prime}}=\left.D^{\alpha} f\right|_{\Omega^{\prime}}, \quad|\alpha| \leq m-1,
$$

and

$$
\left\|\nabla^{m} f\right\|_{L^{p}\left(\Omega^{\prime}\right)} \leq c a_{F} .
$$


Remark 4.2. Corollary 3.2 reads for Whitney jets as follows. Let $\Omega$ be a connected open set in $\mathrm{R}^{n}$. Suppose that

$$
\int_{\Omega} \int_{\Omega} \frac{\left|R^{m-1} F(x, y)\right|^{p}}{|x-y|^{m p+n}} d x d y<\infty .
$$

Then $f$ is a polynomial of degree at most $m-1$ a.e. on $\Omega$.

To prove the theorem we use the sketch of the proof of Theorem 9.1 from [1]. First we show that the next statement is true.

Lemma 4.3. Let $\Omega$ be an open set in $\mathrm{R}^{n}, 1<p<\infty$ and $m$ be a positive integer. Suppose that $(m-1)$-jet $F$ satisfies condition (28). Then for every $\Omega^{\prime} \Subset \Omega$ we have

$$
\lim _{\varepsilon \rightarrow 0} \frac{1}{\varepsilon^{(m-|j|) p}} \int_{\Omega^{\prime}} f_{B(x, \varepsilon)}\left|R_{j}^{m-1-|j|} F(x, y)\right|^{p} d y d x \leq c a_{F},
$$

whenever $|j| \leq m-1$.

Proof. Let $\Omega^{\prime} \Subset \Omega$ and $0<\varepsilon<\operatorname{dist}\left(\Omega^{\prime}, \partial \Omega\right)$. Fix $x, y \in \Omega^{\prime},|x-y|<\varepsilon$. Using Taylor algebra arguments, we have

$$
\begin{aligned}
R_{j}^{m-1-|j|} F(x, y) & =D_{z}^{j}\left[R^{m-1} F(z, x)-R^{m-1} F(z, y)\right]_{z=x} \\
& =D_{z}^{j} P(z ; x, y)_{z=x},
\end{aligned}
$$

where $P(z ; x, y)$ is a polynomial in $z$ of order at most $m-1$.

Since $\varepsilon<\operatorname{dist}\left(\Omega^{\prime}, \partial \Omega\right)$ the set $S=B(x, \varepsilon) \cap B(y, \varepsilon) \subset \Omega$. It is easy to see that

$$
c|S| \geq|B(x, \varepsilon)|=|B(y, \varepsilon)|
$$

for some constant $c$ which is independent of $\varepsilon$. By Markov's inequality [10] applied to the subset $S$ of the ball $B(x, \varepsilon)$, we obtain

$$
\left|D_{z}^{j} P(z ; x, y)\right|_{z=x} \leq \frac{c(n)}{\varepsilon^{|j|}}\left(f_{S}\left|P\left(x^{\prime} ; x, y\right)\right|^{p} d x^{\prime}\right)^{1 / p} .
$$

Thus, from (30), we have

$$
\begin{aligned}
\left|R_{j}^{m-1-|j|} F(x, y)\right| \leq \frac{c(n)}{\varepsilon^{|j|}} & {\left[\left(f_{S}\left|R^{m-1} F\left(x^{\prime}, x\right)\right|^{p} d x^{\prime}\right)^{1 / p}\right.} \\
& \left.+\left(f_{S}\left|R^{m-1} F\left(x^{\prime}, y\right)\right|^{p} d x^{\prime}\right)^{1 / p}\right] \\
= & \frac{c(n)}{\varepsilon^{|i|}}\left[I(x)^{1 / p}+I(y)^{1 / p}\right] .
\end{aligned}
$$


It is easy to see that

$$
\begin{aligned}
\int_{\Omega^{\prime}} f_{B(x, \varepsilon)} I(x) d y d x & \leq c \int_{\Omega^{\prime}} f_{B(x, \varepsilon)} f_{B(x, \varepsilon)}\left|R^{m-1} F\left(x^{\prime}, x\right)\right|^{p} d x^{\prime} d y d x \\
& \leq c \iint_{\left\{\left(x, x^{\prime}\right) \in \Omega:\left|x-x^{\prime}\right|<\varepsilon\right\}}\left|R^{m-1} F\left(x^{\prime}, x\right)\right|^{p} d x^{\prime} d x .
\end{aligned}
$$

On the other hand, we obtain a similar estimate for

$$
\int_{\Omega^{\prime}} f_{B(x, \varepsilon)} I(y) d y d x
$$

which together with (31) proves the claim.

REMARK 4.4. Since (29) implies

$$
\frac{1}{\varepsilon^{n+(m-|j|) p}} \iint_{\left\{(x, y) \in \Omega^{\prime}:|x-y|<\varepsilon\right\}}\left|R_{j}^{m-1-|j|} F(x, y)\right|^{p} d x d y \leq c a_{F},
$$

as $\varepsilon \rightarrow 0,|j| \leq m-1$, using the terminology of Jonsson and Wallin (see e.g. [13]) we can formulate Lemma 4.3 in the following way: If $(m-1)$-jet $F$ satisfies condition (28) then for every $\Omega^{\prime} \Subset \Omega$ we have $F \in \operatorname{Lip}\left(m, p, \infty, \Omega^{\prime}\right)$.

Proof of Theorem 4.1. Let $\Omega^{\prime}$ be an open set such that $\Omega^{\prime} \Subset \Omega$. Decompose $\Omega^{\prime}$ into dyadic cubes. More precisely, let $\mathscr{M}_{k}$ denote a net with mesh $2^{-k}$ in $\mathrm{R}^{n}$ i.e. $\mathscr{M}_{k}$ is a division of $\mathrm{R}^{n}$ into equally large closed cubes with side lenghts $2^{-k}$, obtained by slicing $\mathrm{R}^{n}$ with hyperplanes orthogonal to the coordinate axis.

Set

$$
\Omega_{k}^{\prime}=\left\{x \in \Omega^{\prime}: \operatorname{dist}\left(x, \Omega^{\prime c}\right)>\sqrt{n} 2^{-k+1}\right\}
$$

and

$$
\Omega_{l}^{\prime}=\left\{x \in \Omega^{\prime}: \sqrt{n} 2^{-l+1}<\operatorname{dist}\left(x, \Omega^{\prime c}\right) \leq \sqrt{n} 2^{-l+2}\right\},
$$

for $l=k+1, \ldots$ Define

$$
\mathscr{F}_{k}^{0}=\bigcup_{l=k}^{\infty}\left\{Q \in \mathscr{M}_{l}: Q \cap \Omega_{l}^{\prime} \neq \emptyset\right\}
$$

and denote by $\mathscr{F}_{k}$ the collection of maximal cubes of $\mathscr{F}_{k}^{0}$ (see e.g. [23] for details on the Whitney decomposition).

Then, for each $k$ the collection of cubes $\mathscr{F}_{k}=\left\{Q_{i}^{k}\right\}_{i \in I_{k}}$ satisfies the conditions:

(i) $\Omega^{\prime}=\bigcup_{i \in I_{k}} Q_{i}^{k}$; 
(ii) diam $Q_{i}^{k} \leq 2^{-k} \sqrt{n}$ for all $i \in I_{k}$, moreover,

$$
\operatorname{diam} Q_{i}^{k} \leq \operatorname{dist}\left(Q_{i}^{k}, \Omega^{\prime c}\right) \leq 4 \operatorname{diam} Q_{i}^{k},
$$

if diam $Q_{i}^{k} \leq 2^{-k-1} \sqrt{n}$

(iii) the interiors of the cubes $Q_{i}^{k}$ are pairwise disjoint.

It is easy to see that theses properties imply the next statements as well:

(iv) $\sum_{i \in I_{k}} \chi_{\frac{9}{8}} Q_{i}^{k} \leq c$, where constant $c$ depends only on $n$;

(v) if $Q, K \in \mathscr{F}_{k}$ and $\frac{9}{8} Q \cap \frac{9}{8} K \neq \emptyset$, then $\operatorname{diam} Q \leq \operatorname{diam} K \leq 4 \operatorname{diam} Q$.

Let now $\left\{\varphi_{i}^{k}: i \in I_{k}\right\}$ be a smooth partition of unity subordinated to the decomposition $\mathscr{F}_{k}$. It's standard properties (see [23]) are:

(i) $\operatorname{supp} \varphi_{i}^{k} \subset \mathscr{Q}_{k}^{i}=\frac{9}{8} Q_{i}^{k}$,

(ii) $\sum_{i \in I_{k}} \varphi_{i}^{k} \equiv 1$ on $\Omega^{\prime}$ and

(iii) $\left|D^{\alpha} \varphi_{i}^{k}\right| \leq c_{\alpha}\left(\operatorname{diam} Q_{i}^{k}\right)^{-\alpha}$ in $\mathrm{R}^{n}$.

Define the approximating family

$$
w^{k}(x)=\sum_{i \in I_{k}} \varphi_{i}^{k}(x) T_{\mathscr{Q}_{i}^{k}}^{m-1} F(x),
$$

where $x \in \Omega^{\prime}$ and

$$
T_{Q}^{m-1} F(x)=f_{Q} T_{y}^{m-1} F(x) d y .
$$

Let $x$ be in $\Omega^{\prime}$, denote by $B_{x}^{k}$ the ball $B\left(x, r_{k}\right)$,

$$
r_{k}=\sqrt{n} \max \left\{\operatorname{diam} \mathscr{Q}_{i}^{k}: x \in \mathscr{Q}_{i}^{k}\right\} .
$$

By property (v) of cubes $Q_{i}^{k}$ the radius $r_{k} \approx \operatorname{diam} Q_{i}^{k}$ whenever $x \in Q_{i}^{k}$ and, evidently, $\mathscr{Q}_{i}^{k} \subset B_{x}^{k}$ for every $i$ such that $x \in \mathcal{Q}_{i}^{k}$. Consider

$$
\begin{aligned}
\left|f_{0}(x)-w^{k}(x)\right| & =\left|\sum_{i \in I_{k}} \varphi_{i}^{k}(x) f_{\mathscr{Q}_{i}^{k}}\left[f_{0}(x)-T_{y}^{m-1} F(x)\right] d y\right| \\
& \leq \sum_{i \in I_{k}} \varphi_{i}^{k}(x) f_{\mathscr{Q}_{i}^{k}}\left|R^{m-1} F(x, y)\right| d y \chi_{\mathscr{Q}_{i}^{k}}(x) \\
& \leq c \sum_{i \in I_{k}} \varphi_{i}^{k}(x) f_{B_{x}^{k}}\left|R^{m-1} F(x, y)\right| d y \\
& =c f_{B_{x}^{k}}\left|R^{m-1} F(x, y)\right| d y .
\end{aligned}
$$


Thus,

$$
\left\|f_{0}-w^{k}\right\|_{L^{p}\left(\Omega^{\prime}\right)} \leq c\left(\int_{\Omega^{\prime}} f_{B_{x}^{k}}\left|R^{m-1} F(x, y)\right|^{p} d y d x\right)^{1 / p} \leq c 2^{-k m},
$$

as $k \rightarrow \infty$, which shows that the sequence of functions $w^{k}$ converges in $L^{p}\left(\Omega^{\prime}\right)$ to $f_{0}$.

Now let $|\alpha|=m$ and consider

$$
\begin{aligned}
D^{\alpha} w^{k}(x) & =\sum_{i} D^{\alpha}\left(\varphi_{i}^{k}(x) T_{\mathscr{Q}_{i}^{k}}^{m-1} F(x)\right) \\
& =\sum_{i} \sum_{\beta \leq \alpha} \frac{\alpha !}{\beta !(\alpha-\beta) !} D^{\beta} \varphi_{i}^{k}(x) D^{\alpha-\beta}\left(T_{\mathscr{Q}_{i}^{k}}^{m-1} F\right)(x) \\
& =\sum_{\substack{\beta+\gamma=\alpha \\
|\gamma| \leq m-1}} \frac{\alpha !}{\beta !(\alpha-\beta) !} S_{\beta, \gamma}(x),
\end{aligned}
$$

where

$$
S_{\beta, \gamma}(x)=\sum_{i} D^{\beta} \varphi_{i}^{k}(x) T_{\mathscr{Q}_{i}^{k}}^{m-1-|\gamma|} D^{\gamma} F(x)
$$

Since $\sum_{i} D^{\beta} \varphi_{i}(x) \equiv 0$ if $|\beta| \geq 1$,

$$
\begin{aligned}
S_{\beta, \gamma}(x) & =S_{\beta, \gamma}^{\prime}(x) \\
& \equiv \sum_{i} D^{\beta} \varphi_{i}^{k}(x)\left[f_{\gamma}(x)-T_{2_{i}^{k}}^{m-1-|\gamma|} D^{\gamma} F(x)\right] \\
& =\sum_{i} D^{\beta} \varphi_{i}^{k}(x) f_{2_{i}^{k}} R_{\gamma}^{m-1-|\gamma|} F(x, y) d y
\end{aligned}
$$

and

$$
\left|S_{\beta, \gamma}^{\prime}(x)\right| \leq \sum_{i}\left|D^{\beta} \varphi_{i}^{k}(x)\right| f_{B_{x}^{k}}\left|R_{\gamma}^{m-1-|\gamma|} F(x, y)\right| d y \chi_{Q_{i}^{k}}(x) .
$$

By smoothness properties of the partition of unity

$$
\left|D^{\beta} \varphi_{i}^{k}(x)\right| \leq c\left(\operatorname{diam} Q_{i}^{k}\right)^{-|\beta|} \leq c 2^{k|\beta|},
$$

for every $x \in \mathcal{Q}_{i}^{k}$. Hence, the $L^{p}$-norm of $S_{\beta, \gamma}^{\prime}$ can be estimated in the following 
way

$$
\begin{aligned}
\left\|S_{\beta, \gamma}^{\prime}\right\|_{L^{p}\left(\Omega^{\prime}\right)}^{p} & =\int_{\Omega^{\prime}}\left(\sum_{i}\left|D^{\beta} \varphi_{i}^{k}(x)\right| f_{B_{x}^{k}}\left|R_{\gamma}^{m-1-|\gamma|} F(x, y)\right| d y \chi_{2_{i}^{k}}(x)\right)^{p} d x \\
& \leq c 2^{k p|\beta|} \int_{\Omega^{\prime}}\left(f_{B_{x}^{k}}\left|R_{\gamma}^{m-1-|\gamma|} F(x, y)\right| d y\right)^{p} d x \\
& \leq c 2^{k p|\beta|} \int_{\Omega^{\prime}} f_{B_{x}^{k}}\left|R_{\gamma}^{m-1-|\gamma|} F(x, y)\right|^{p} d y d x .
\end{aligned}
$$

Thus, by Lemma 4.3 for $|\alpha|=m$ and large $k$ we have

$$
\left\|D^{\alpha} w^{k}\right\|_{L^{p}\left(\Omega^{\prime}\right)} \leq c a_{F} 2^{(|\beta|+|\gamma|-m) k} \leq c a_{F}
$$

(one could set $\varepsilon=2^{-k}$ in (29)).

We show that for $|\alpha| \leq m-1$ the derivatives $D^{\alpha} w^{k}$ converge in $L^{p}\left(\Omega^{\prime}\right)$ to the elements $f_{\alpha}$ of the jet $F$. A computation shows that

$$
\begin{aligned}
f_{\alpha}(x)-D^{\alpha} w^{k}(x)= & \sum_{i} \varphi_{i}^{k}(x) f_{\alpha}(x)-\sum_{i} D^{\alpha}\left(\varphi_{i}^{k}(x) T_{\mathscr{Q}_{i}^{k}}^{m-1} F(x)\right) \\
= & \sum_{i} \varphi_{i}^{k}(x)\left(f_{\alpha}(x)-T_{\mathscr{Q}_{i}^{k}}^{m-1-|\alpha|} D^{\alpha} F(x)\right) \\
& \quad+\sum_{\substack{\beta+\gamma=\alpha \\
|\beta|>0}} \frac{\alpha !}{\beta !(\alpha-\beta) !} \sum_{i} D^{\beta} \varphi_{i}^{k}(x) T_{\mathscr{Q}_{i}^{k}}^{m-1-|\gamma|} D^{\gamma} F(x) \\
= & \sum_{\beta+\gamma=\alpha} \frac{\alpha !}{\beta !(\alpha-\beta) !} S_{\beta, \gamma}^{\prime}(x) .
\end{aligned}
$$

Using the above estimate for $\left\|S_{\beta, \gamma}^{\prime}\right\|_{L^{p}\left(\Omega^{\prime}\right)}^{p}$, we arrive at

$$
\left\|f_{\alpha}-D^{\alpha} w^{k}\right\|_{L^{p}\left(\Omega^{\prime}\right)} \leq c 2^{(|\beta|+|\gamma|-m) k}=c 2^{(|\alpha|-m) k} \rightarrow 0,
$$

as $k \rightarrow \infty$.

Thus, we have shown that the sequence of smooth functions $w^{k}$ converges to function $f_{0}$ in $L^{p}\left(\Omega^{\prime}\right)$ and for any $\alpha,|\alpha| \leq m$, the sequence of derivatives $D^{\alpha} w^{k}$ is bounded in $L^{p}\left(\Omega^{\prime}\right)$. Since $p>1$, by weak compactness argument it follows that $f_{0} \in W^{m, p}\left(\Omega^{\prime}\right)$.

Let $f \equiv f_{0}$, then by (34) $\left.D^{\alpha} f\right|_{\Omega^{\prime}}=\left.f_{\alpha}\right|_{\Omega^{\prime}},|\alpha| \leq m-1$, and by (33) we have estimate $\left\|\nabla f^{m}\right\|_{L^{p}\left(\Omega^{\prime}\right)} \leq c a_{F}$.

REMARK 4.5. We can also consider the meaning of the condition (28) for the Whitney jet on an $s$-set in $\mathrm{R}^{n}$. Recall that a subset $S \subset \mathrm{R}^{n}$ is called an $s$-set 
(or Ahlfors $s$-regular) if there are constants $c_{1}, c_{2}>0$ such that for every ball $B=B(x, r)$ with center at $S$ and $r \leq \operatorname{diam} S$

$$
c_{1} r^{s} \leq H^{s}(B(x, r) \cap S) \leq c_{2} r^{s},
$$

where $H^{s}$ denote the $s$-dimensional Hausdorff measure on $\mathrm{R}^{n}$.

Let $S$ be an $s$-set with $n-1<s \leq n$. Consider a $(m-1)$-jet

$$
F=\left\{f_{\alpha}:|\alpha| \leq m-1\right\}
$$

$f_{\alpha} \in L^{p}\left(S, H^{s}\right)$, such that it's formal Taylor remainder of order $m-1$ satisfies the condition

$$
\frac{1}{\varepsilon^{s+m p}} \iint_{\{(x, y) \in S:|x-y|<\varepsilon\}}\left|R^{m-1} F(x, y)\right|^{p} d H^{s} d H^{s} \leq a_{F}
$$

as $\varepsilon \rightarrow 0$. Then the functions $f_{\alpha}$ are uniquely determined by $f_{0}$. The proof uses slightly modified Lemma 4.3 and arguments as in Theorem 2 in [13], p. 126. Thus, if $f_{0}$ is identically zero then $f_{\alpha}$ are zero as well and we can identify the jet $F$ with the single function $f_{0}$.

In particular, if $\Omega$ is a $W^{m, p}$-extension domain then $\Omega$ is an $n$-set (see [14]) and the statement "a jet $F$ satisfies (35) on $\Omega$ " is equivalent to the fact that $f_{0} \in W^{m, p}(\Omega)$. If $S$ is an arbitrary $s$-set with $n-1<s \leq n$ then condition (35) characterizes the Lipschitz space $\operatorname{Lip}(m, p, \infty, S)$ introduced by A. Jonsson and H. Wallin [13].

The first order spaces $\operatorname{Lip}(1, p, \infty, S)$ and their relations to Sobolev spaces on metric measure spaces were studied in [25]. For instance, from the results in [25] it follows that if $S$ supports the $q$-Poncaré inequality, $1 \leq q<\infty$, then $\operatorname{Lip}(1, p, \infty, S)$ coincides with the Hajłasz-Sobolev space $W^{1, p}(S)$ for $p>q$.

\section{REFERENCES}

1. Bojarski, B., Pointwise characterization of Sobolev classes, Proc. Steklov Inst. Math. 255 (2006), 65-81.

2. Bojarski, B., Remarks on the Bourgain-Brezis-Mironescu approach to Sobolev spaces, Bull. Pol. Acad. Sci. Math. 59 (2011), 65-75.

3. Bojarski, B., Hajłasz, P., and Strzelecki, P., Improved $C^{k, \lambda}$ approximation of higher order Sobolev functions in norm and capacity, Indiana Univ. Math. J. 51 (2002), 507-540.

4. Borghol, R., Some properties of Sobolev spaces. Asymptot. Anal. 51 (2007), 303-318.

5. Bourgain, J., Brézis, H., and Mironescu, P., Another look at Sobolev spaces, pp. 439-455 in: Optimal Control and Partial Differential Equations, IOS Press, Amsterdam 2001.

6. Bourgain, J., and Nguyen, H.-M., A new characterization of Sobolev spaces, C. R. Acad. Sci. Paris 343 (2006), 75-80. 
7. Brézis, H., How to recognize constant functions. A connection with Sobolev spaces, Uspekhi Mat. Nauk 57 (2002), 59-74; transl. Russian Math. Surveys 57 (2002), 693-708.

8. Brézis, H., and Nguyen, H.-M., On a new class of functions related to VMO, C. R. Math. Acad. Sci. Paris 349 (2011), 157-160.

9. Calderón, A. P., Estimates for singular integral operators in terms of maximal functions, Studia Math. 44 (1972), 563-582.

10. Campanato, S., Proprietà di una famiglia di spazi funzionali, Ann. Scuola Norm. Sup. Pisa 18 (1964), 137-160.

11. Dávila, J., On an open question about functions of bounded variation, Calc. Var. Partial Differential Equations 15 (2002), 519-527.

12. DeVore, R. A., and Sharpley, R. C., Maximal functions measuring smoothness, Mem. Amer. Math. Soc. 47, (1984), no. 293.

13. Jonsson, A., and Wallin, H., Function spaces on subsets of $\mathrm{R}^{n}$, Math. Rep. 2 (1984), no. 1.

14. Hajłasz, P., Koskela, P., and Tuominen, H., Sobolev embeddings, extensions and measure density condition, J. Funct. Anal. 254 (2008), 1217-1234.

15. Maz’ya, V. G., Sobolev spaces, Springer, Berlin, 1985.

16. Nguyen, H.-M., Some new characterizations of Sobolev spaces, J. Funct. Anal. 237 (2006), 689-720.

17. Nguyen, H.-M., Further characterizations of Sobolev spaces, J. Eur. Math. Soc. 10 (2008), 191-229.

18. Nguyen, H.-M., Г-convergence, Sobolev norms, and BV functions, Duke Math. J. 157 (2011), 495-533.

19. Nguyen, H.-M., Some inequalities related to Sobolev norms, Calc. Var. Partial Differential Equations 41 (2011), 483-509.

20. Pietruska-Pałuba, K., Heat kernels on metric spaces and a characterisation of constant functions, Manuscripta Math. 115 (2004), 389-399.

21. Ponce, A., A new approach to Sobolev spaces and connections to $\Gamma$-convergence, Calc. Var. Partial Differential Equations 19 (2004), 229-255.

22. Ponce, A., An estimate in the spirit of Poincaré's inequality, J. Eur. Math. Soc. 6 (2004), 1-15.

23. Stein, E. M., Singular Integrals and Differentiability Properties of Functions, Princeton Math. Ser. 30, Princeton Univ. Press, Princeton 1970.

24. Whitney, H., Analytic extensions of differentiable functions defined in closed sets, Trans. Amer. Math. Soc. 36 (1934), 63-89.

25. Yang, D., and Lin, Y., Spaces of Lipschitz type on metric spaces and their applications, Proc. Edinburgh Math. Soc. (2) 47 (2004), 709-752.

26. Ziemer, W. P., Weakly differentiable functions. Sobolev spaces and functions of bounded variation, Grad. Texts Math. 120, Springer, Berlin 1989.

INSTITUTE OF MATHEMATICS

POLISH ACADEMY OF SCIENCES

00-956 WARSAW

POLAND

E-mail: b.bojarski@impan.gov.pl
DEPARTMENT OF MATHEMATICS

P.O. BOX 11100

FI-00076 AALTO UNIVERSITY

FINLAND

E-mail: lizaveta.ihnatsyeva@aalto.fi juha.k.kinnunen@aalto.fi 